\title{
ON THE CONVOL.UTION OF A MEASURE AND A FUNCTION
}

\author{
S. K. BERBERIAN
}

ABSTRACT. Complements to a theorem of Bourbaki on the convolution of a measure and a function.

The setting is as follows [3, Chapter VIII, $\$ 4$, No. 1]: $X$ is a locally compact space, $G$ is a locally compact group acting continuously on the left in $X$, and $\beta$ is a nonzero positive measure on $X$ that is quasi-invariant under $G$; more precisely,

$$
\gamma(s) \beta=\chi^{\left(s^{-1}, \cdot\right) \cdot \beta}
$$

for all $s \in G$, where $\chi$ is universally measurable and everywhere $>0$ on $G \times X$.

The following proposition is central to the discussion of convolution of functions in [3]:

Proposition [3, Chapter VIII, \$4, No. 1, Proposition 2]. Let $\mu$ be a measure on $G$, $f$ a locally $\beta$-integrable complex function on $X$. Assume that one of the following conditions is verified:

(i) $f$ and $x$ are continuous;

(ii) $G$ operates properly in $X$, and $f$ is zero on the complement of a denumerable union of compact sets;

(iii) $\mu$ is carried by a denumerable union of compact sets.

If $\mu$ and $f$ are convolvable relative to $\beta$, then the function $s \mapsto f\left(s^{-1} x\right)$ $\chi^{\left(s^{-1}, x\right)}$ is essentially $\mu$-integrable for locally $\beta$-almost all $x$; and if $\mu * f$ denotes any locally $\beta$-integrable function such that $(\mu * f) \cdot \beta=\mu *(f \cdot \beta)$, then

$$
(\mu * f)(x)=\int f\left(s^{-1} x\right) \chi\left(s^{-1}, x\right) d \mu(s)
$$

locally $\beta$-almost everywhere.

Case (ii) apparently needs an additional hypothesis, and the proof of Case (iii) given in [3] has some sizable gaps. The aim of this paper is to clarify these points; also, we reformulate condition (iii) so as to make it more flexible in applications, one of which is given. All notations and terminology are taken from [1]-[3].

By way of motivation, we remark that the roughly comparable result in

Received by the editors October 21, 1974.

AMS (MOS) subject clas sifications (1970). Primary $28 A 70$. 
the treatise of E. Hewitt and K. A. Ross is Lemma (20.6) of [5]. There, the function $f$ is assumed to be a Borel function, therefore the function $(s, x)$ $\mapsto f\left(s^{-1} x\right)$ is a Borel function on the product space. This smoothes the way for an application of Fubini's theorem [5, Theorem (13.9)]; moreover, all of the partial functions $s \mapsto f\left(s^{-1} x\right), x \mapsto f\left(s^{-1} x\right)$ are Borel functions. Consequently, the measurability problem considered in Lemma 3 below does not arise in [5]; on the other hand, the Bourbaki formulation is more flexible in that non-Borel functions are allowed.

Lemma 1. The following conditions on a measure $\mu$ are equivalent:

(a) $\mu$ is carried by a denumerable union of compact sets;

(b) $\mu$ is carried by a denumerable union of essentially $\mu$-integrable sets;

(c) $\mu$ is carried by a $\mu$-moderated, $\mu$-measurable set;

(d) $|\mu|=\Sigma_{1}^{\infty} \mu_{n}$, where $\left(\mu_{n}\right)$ is a summable sequence of bounded positive measures.

Proof. We can suppose $\mu \geq 0$. It is trivial that (a) implies (b).

(b) $\Rightarrow(d)$. Suppose $\mu$ is carried by $S=\bigcup_{1}^{\infty} S_{n}$, where the $S_{n}$ are essentially $\mu$-integrable and, as we may suppose, disjoint. The measures $\mu_{n}=$ $\phi_{S_{n}} \cdot \mu$ ( $\phi$ denotes characteristic function) are bounded $[2, S 5$, No. 3 , Corollary of Theorem 1]; since $\mu=\phi_{S} \cdot \mu$ and $\phi_{S}=\Sigma_{1}^{\infty} \phi_{S_{n}}$, it is an elementary consequence of the Lebesgue dominated convergence theorem that $\mu=\Sigma_{1}^{\infty} \mu_{n}$ [1, Chapter IV, $\{4$, No. 3, Corollary 2 of Theorem 2], [2, $\$ 2$, No. 1].

(d) $\Rightarrow$ (c). Suppose $\mu=\Sigma_{1}^{\infty} \mu_{n}$, where the $\mu_{n}$ are bounded positive measures. For each $n$, write $\mu_{n}=f_{n} \cdot \mu$ with $f_{n}$ essentially $\mu$-integrable $[2, \$ 5$, No. 5, Theorem 2 and No. 3, Corollary of Theorem 1]. We can suppose that $f_{n}$ is $\mu$-integrable [2, $\S 5$, No. 3, Corollary 2 of Proposition 3]. Then for every $n$, the set $A_{n}=\left\{s: f_{n}(s) \neq 0\right\}$ is $\mu$-measurable, and $\mu$-moderated $[2, \delta 1$, No. 3 , Corollary of Proposition 9], hence so is $A=\bigcup_{1}^{\infty} A_{n}$. For all $n$, evidently $\phi_{A} \cdot \mu_{n}=\mu_{n}$, that is, CA is locally $\mu_{n}$-negligible; it follows that CA is locally $\mu$-negligible [2, $\$ 2$, No. 2, Corollary 2 of Proposition 1], in other words $A$ carries $\mu$.

(c) $\Rightarrow($ a). Suppose $A$ is a $\mu$-moderated, $\mu$-measurable set such that $\phi_{A} \cdot \mu=\mu$. Then $\Lambda \subset \bigcup_{1}^{\infty} K_{n} \cup N$, where the $K_{n}$ are compact and $N$ is $\mu$-negligible. Writing $S=\bigcup_{1}^{\infty} K_{n}$, the relation CS $\subset N \cup \mathrm{C} A$ shows that $\mathrm{CS}$ is locally $\mu$-negligible.

Lemma 2. If $f$ is a locally $\beta$-integrable function on $X$ and if $\mu$ is a measure on $G$ such that $\mu$ and $f$ are convolvable relative to $\beta$, then the function $F(s, x)=f\left(s^{-1} x\right) \chi\left(s^{-1}, x\right)$ on $G \times X$ is measurable for $\mu \otimes \beta$.

Proof. For every $h \in \mathcal{K}(X)$, the function $(1 \otimes b) F$ is essentially integrable for $\mu \otimes \beta[3$, Chapter VIII, $\S 4$, No. 1, proof of Proposition 2], hence 
$\mu \otimes \beta$-measurable. Then $F$ is $\mu \otimes \beta$-measurable by a routine application of the principle of localization [1, Chapter IV, $\$ 5$, No. 2, Proposition 4]. Incidentally, in view of the hypotheses on $\chi$, it is the same to say that the function $(s, x) \mapsto f\left(s^{-1} x\right)$ is $\mu \otimes \beta$-measurable.

Lemma 3. With hypotheses as in Lemma 2, let $M=\{x: F(\cdot, x)$ is not $\mu$-measurable $\}$.

(1) If $f$ and $\chi$ are continuous, then $M=\varnothing$.

(2) If $F$ (equivalently, the function $(s, x) \mapsto f\left(s^{-1} x\right)$ ) is moderated for $\mu \otimes \beta$, then $M$ is $\beta$-negligible.

(3) If $\mu$ is carried by a denumerable union of compact sets, then $M$ is locally $\beta$-negligible.

Proof. (1) For every $x, F(\cdot, x)$ is continuous.

(2) In view of Lemma 2 , this is imm ediate from $[2, \S 8$, No. 2, Proposition (2a)].

(3) In view of criterion (d) of Lemma 1, this follows from the proof of $[2, \S 8$, No. 2, Proposition (2b)].

Proof of the Proposition (under an added hypothesis in Case (ii)). We can suppose $f \geq 0$ and $\mu \geq 0$. Let $F(s, x)=f\left(s^{-1} x\right) \chi\left(s^{-1}, x\right)$, and let $g: X$ $\rightarrow \overline{\mathbf{R}}_{+}$be the function defined by the formula $g(x)=\int^{\bullet} F(\cdot, x) d \mu$. One has $g(x)=\int^{*} F(\cdot, x) d \mu$ in Case (i) (because every $F(\cdot, x)$ is continuous $[2, \S 1$, No. 1, Proposition 4]) and in Case (ii) (because, for each $x, F(\cdot, x)$ vanishes outside a denumerable union of compact sets [3, Chapter III, $\$ 4$, No. 5, Theorem (1b)]).

Cases (i), (ii). As shown in [3], $g$ is locally $\beta$-integrable and $g \cdot \beta=$ $\mu *(f \cdot \beta)$, that is, $g$ is a determination of $\mu * f$. In particular, the set $N=$ $\{x: g(x)=+\infty\}$ is locally $\beta$-negligible. In Case (i), this means (in view of part (1) of Lemma 3 ) that $F(\cdot, x)$ is $\mu$-integrable for locally $\beta$-almost all $x$. In Case (ii), if one assumes that the function $(s, x) \mapsto f\left(s^{-1} x\right)$ is $\mu \otimes \beta$. moderated, then it results from part (2) of Lemma 3 that $F(\cdot, x)$ is $\mu$-integrable for locally $\beta$-almost all $x$.

Case (iii). Suppose $\mu$ is carried by a denumerable union $S$ of compact sets. Since $\phi_{S}=1$ locally $\mu$-almost everywhere and $S$ is $\mu$-moderated, one has

$$
g(x)=\int^{\bullet} F(\cdot, x) \phi_{S} d \mu=\int^{*} F(\cdot, x) \phi_{S} d \mu
$$

for all $x \in X$. As shown in [3] $g$ is again locally $\beta$-integrable and is a determination of $\mu * f$. In particular, $g(x)<+\infty$ locally $\beta$-a.e.; in view of part (3) of Lemma 3, this means that $F(\cdot, x)$ is essentially $\mu$-integrable for locally $\beta$-almost all $x[2, \S 1$, No. 3, Proposition 9]. 
The following application is a slight extension of [3, Chapter VIII, $\$ 4$, No. 5, Proposition 10]:

Corollary. Let $\beta$ be a relatively invariant, nonzero positive measure on $G$, and let $f, g$ be locally $\beta$-integrable functions on $G$ such that $f$ and $g$ are convolvable relative to $\beta$. If one of $f, g$ is continuous or is zero outside a denumerable union of essentially $\beta$-integrable sets, then

$$
(f * g)(x)=\int g\left(s^{-1} x\right) f(s) \chi\left(s^{-1}\right) d \beta(s)=\int f\left(x s^{-1}\right) g(s) \chi^{\prime}\left(s^{-1}\right) d \beta(s)
$$

for locally $\beta$-almost all $x$.

Proof. Here $\chi$ and $\chi^{\prime}$ denote the left and right multiplicators of $\beta$, which are continuous [3, Chapter VII, $\$ 1$, No. 8]. It is straightforward to show that the two essential integrals (or integrals) exist simultaneously and are then equal, thus it is immaterial whether the conditions are imposed on $f$ or on $g$. We can suppose $f \geq 0, g \geq 0$. Let $\mu=f \cdot \beta$.

If $g$ is continuous, one applies Case (i) of the Proposition to $\mu, g$.

Suppose $f$ is zero outside $S=\bigcup_{1}^{\infty} A_{n}$, where the $A_{n}$ are essentially $\beta$-integrable. For each $n$, let $h_{n}$ be a $\beta$-integrable function such that $\phi_{A_{n}}=$ $b_{n}$ locally $\beta$-a.e. Let $B_{n}=\left\{x: h_{n}(x)=1\right\}$. From $\phi_{B_{n}}=b_{n} \phi_{B_{n}}$, we see that $B_{n}$ is $\beta$-integrable. Since $h_{n}^{2}=h_{n}$ locally $\beta$-a.e., it results that $h_{n}=\phi_{B_{n}}$ locally $\beta$-a.e. (indeed, $\beta$-a.e. [2, 1 , No. 3, Lemma 1]), therefore $\phi_{A_{n}}=\phi_{B_{n}}$ locally $\beta$-a.e. It follows easily that $S \subset B \cup N$, where $B=\bigcup_{1}^{\infty} B_{n}$ is $\beta$-moderated and $N$ is locally $\beta$-negligible; one can even suppose that $B$ is a denumerable union of compact sets [2, $\$ 1$, No. 2, Proposition 5]. Since $N$ is also locally negligible for $\mu=f \cdot \beta[2, \$ 5$, No. 5, Theorem 2], it follows that $\mu$ is carried by $B$; thus we are in the situation of Case (iii) of the Proposition.

Remark. In the Corollary, it also suffices that one of $f, g$ be equal locally $\beta$-a.e. to a continuous function. More generally, suppose $f, g, f^{\prime}, g^{0}$ are locally $\beta$-integrable functions such that $f=f^{\prime}$ locally $\beta$-a.e. and $g=g^{\prime}$ locally $\beta$-a.e. It is elementary that if $f$ and $g$ are convolvable relative to $\beta$, then so are $f^{\prime}$ and $g^{\prime}$, and one then has $f * g=f^{\prime} * g^{\prime}$ locally $\beta$-a.e. Suppose, in addition, that $f * g$ has a determination $h$ such that $h(x)$, for locally $\beta$-almost all $x$, is given by the (coexisting) integral formulas of the Corollary; for such an $x$, the first formula shows that $f$ may be replaced by $f^{\prime}$ (in both formulas), the second that $g$ may be replaced by $g^{\prime \prime}$; thus $f^{\prime} * g^{\prime}$ is also given by such formulas locally $\beta$-a.e.

\section{REFERENCES}

1. N. Bourbaki, Eléments de mathématique. Fasc. XIII. Livre VI: Intégration. Chaps. I-IV, 2ième éd., Actualités Sci. Indust., no. 1175, Hermann, Paris, 1965. MR $36 \# 2763$. 
2. N. Bourbaki, Éléments de mathématique. Fasc. XXI. Livre VI: Intégration. Chap. V. 2ième éd., Actualités Sci. Indust., no. 1244, Hermann, Paris, 1967. MR 35 \#322.

3. - Éléments de mathématique. Fasc. XXIX. Livre VI: Intégration. Chaps. VII-VIII, Actualifés Sci. Indust., no. 1306, Hermann, Paris, 1963. MR 31 \#3539.

4. - Eléments de mathématique. Fasc. III. Livre III: Topologie générale. Chaps. III-IV, 3ième éd., Actualités Sci. Indust,, no. 1143, Hermann, Paris, 1960.

5. E. Hewitt and K. A. Ross, Abstract harmonic analysis, Vol. I: Structure of topological groups. Integration theory, group representations, Die Grundlehren der math. Wissenschaften, Band 115, Academic Press, New York; Springer-Verlag, Berlin, 1963. MR $28 \# 158$.

DEPARTMENT OF MATHEMATICS, UNIVERSITY OF TEXAS, AUSTIN, TEXAS 78712 